\title{
Stochastic Fluctuations of Low-Energy Cosmic Rays and the Interpretation of Voyager Data
}

\section{Vo Hong Minh Phan, ${ }^{a, b, *}$ Florian Schulze, ${ }^{a}$ Philipp Mertsch, ${ }^{a}$ Sarah Recchia ${ }^{c}$ and Stefano Gabici $^{b}$}

${ }^{a}$ Institute for Theoretical Particle Physics and Cosmology (TTK), RWTH Aachen University, 52056 Aachen, Germany

${ }^{b}$ Université de Paris, CNRS, Astroparticule et Cosmologie, F-75006 Paris, France

${ }^{c}$ Dipartimento di Fisica, Universitá di Torino, via P. Giuria 1, 10125 Torino, Italy

E-mail: vhmphan@physik.rwth-aachen.de,

florian.tobias.schulze@rwth-aachen.de, pmertsch@physik.rwth-aachen.de, sarah.recchia@unito.it, gabici@apc.in2p3.fr

The propagation distances of $\mathrm{MeV}$ cosmic rays are significantly limited due to ionisation loss in the interstellar medium. Therefore, the density of cosmic rays will depend sensitively on the exact distribution of the sources in space and time. For an ensemble of source distributions, the fluxes will follow a strongly non-Gaussian distribution. Here, we show that the typical flux, that is the median of the distribution, significantly deviates from the average flux, that is the expectation value of the distribution. Taking this into account allows for a consistent fit of data from Voyager 1 and AMS-02 without any unmotivated breaks in the source spectrum or mean-free path where earlier models needed to introduce those in an ad hoc fashion. We conclude with a discussion of the implication of these results for the discrepancy between the observed and predicted ionization rate induced by low-energy cosmic rays.

$37^{\text {th }}$ International Cosmic Ray Conference (ICRC 2021)

July 12th-23rd, 2021

Online - Berlin, Germany

\footnotetext{
*Presenter
} 


\section{Introduction}

Despite over 100 years of intense experimental and theoretical efforts, the origin of Galactic cosmic rays (GCRs) has still not been unambiguously identified. At energies above a few tens of $\mathrm{GeV}$, much progress has been made in the last couple of years, thanks to direct observations by high-precision, high-statistics experiments like AMS-02 or PAMELA and the study of gammarays by Fermi-LAT and Cherenkov telescopes [1]. At lower energies, however, the situation is still very much unclear since the intensities of GCRs would suffer significant spatial and temporal modifications as they interact with the solar wind [2]. In 2013, however, the first direct observations of interstellar spectra by Voyager 1 were published and it became clear that simple extrapolations from higher energies fail [3]. Specifically, in order to fit both Voyager 1 and AMS-02 data, simple diffusive transport models have to introduce breaks in the source spectra around a GeV in an ad hoc fashion, while the physical interpretation of such a break is rather questionable [4]. In fact, we would maintain that no convincing explanation of such a break has been put forward to date.

An important effect for MeV GCRs that has been ignored in the literature is due to the discrete nature of sources. Instead, the distribution of sources in position and time is oftentimes modelled as smooth. Even though the sources are likely separate, discrete objects like supernova remnants (SNRs), this approximation is admissible at $\mathrm{GeV}$ energies, since the transport distances and times exceed the typical source separations and ages. However, if energy losses reduce the propagation times and distances, this approximation breaks down and instead the discrete nature of the sources needs to be taken into account. An example where this approach has been followed are high-energy electrons and positrons at hundreds of $\mathrm{GeV}$ and above, which lose energy due to the synchrotron and inverse Compton processes [5], but ionisation losses also severely limit the propagation of $\mathrm{MeV}$ GCRs. Predicting their local intensities therefore requires rather precise knowledge of the ages and distances of the sources. While some young and nearby sources might be known, catalogues of such sources remain necessarily incomplete, in particular with respect to far away and old sources.

Instead, the distribution of sources can be considered a statistical ensemble, thus opening the path towards a statistical modelling of GCR intensities. Operationally, one draws a set of source distances and ages from the statistical probability density function (PDF). Adding up their intensities results in a prediction for this given realisation of the sources. Repeating this procedure for a large number of realisations, one can estimate the distribution of intensities. The first moment and second central moment of this distribution are the expectation value and the variance. Since the expectation value $\langle\psi\rangle$ could be obtained by averaging over many realizations, it approaches the solution of the GCR transport equationwhen the smooth source PDF, from which individual source distance and ages are drawn, is used as the source term $q$. However, as it turns out the statistics of the intensities is markedly non-Gaussian, with the second moment divergent. This is due to the long power law tails of the intensity PDF. Its asymmetric shape renders the expectation value different from the median and from the maximum of the distribution [6].

In the following, we model the intensities of GCR protons and electrons between $1 \mathrm{MeV}$ and $10 \mathrm{GeV}$ taking into account the stochasticity induced by the discreteness of sources. Consequently, our predictions will be probabilistic. We will illustrate that the expectation value is a bad estimator for the intensities in individual realisations. For instance, for low enough energies the expectation value is outside the $68 \%$ uncertainty band. Furthermore, its spectral shape is markedly different 
than the intensity in any individual realisation. Finally, we stress that the expectation value does not reproduce the data either unless an artificial break is added to the source spectrum. Instead we suggest considering the median of the intensity PDF as a better measure of what a "typical" intensity will look like, and the reference intensity around which the intensities from all realisations are distributed. Interestingly, the data for protons and electrons fall squarely within the uncertainty bands. We thus conclude that a model without artificial breaks is to be preferred in explaining the Voyager and AMS-02 data as long as the stochasticity effect is taken into account.

\section{Modelling}

The cosmic ray density $\psi$ at an arbitrary position ( $r$ and $z$ ), energy $E$ and time $t$ with contributions from all individual sources could be obtained by solving the following transport equation,

$$
\frac{\partial \psi}{\partial t}+\frac{\partial}{\partial z}(u \psi)-D \nabla^{2} \psi+\frac{\partial}{\partial E}(\dot{E} \psi)=\sum_{i=1}^{N_{\mathrm{s}}} Q(E) \frac{\delta\left(r-r_{i}\right)}{2 \pi r_{i}} \delta\left(z-z_{i}\right) \delta\left(t-t_{i}\right),
$$

where $u=u(z)$ is the advection velocity profile with only the component perpendicular to the Galactic disk, $D=D(E)$ is the isotropic and homogeneous diffusion coefficient, $\dot{E}$ describes the energy loss rate for GCRs both inside the Galactic disk and in the magnetized halo, the sum on the RHS represents the contribution from sources of different distances and ages, $Q(E)$ denotes the spectrum that an individual source injects into the ISM. The total intensity from $N_{\mathrm{s}}$ sources is then just the sum over the Green's function $\mathcal{G}\left(r, z, E ; r_{i}, z_{i}, t-t_{i}\right)$ of Eq. (1) at the position of the solar system,

$$
\psi=\sum_{i} \mathcal{G}\left(r=0, z=z_{\odot}, E ; r_{i}, z_{i}, t-t_{i}\right) .
$$

where $z=z_{\odot} \simeq 14 \mathrm{pc}$ is the vertical offset of the solar system from the Galactic mid-plane [7].

Equation 1 is solved numerically assuming GCRs propagate within a finite cylindrical region with height $2 L \simeq 8 \mathrm{kpc}$ and radius $r_{\max } \simeq 10 \mathrm{kpc}$ centering around the source. The other parameters of our model are chosen such that the most probable values of the intensity is compatible with the observational data (see [8] and references therein for more details on the externally constrained and fitted parameters). Specifically, the advection velocity is assumed to have the following profile $u(z)=u_{0} \operatorname{sgn}(z)$ with $u_{0}=16 \mathrm{~km} / \mathrm{s}$, where $\operatorname{sgn}(z)$ is the sign function. We assume also the diffusion coefficient of the form suggested in [9] which is $D(E) \sim \beta \gamma^{\delta}$ where $\beta=v / c$ is the ratio between the particles' speed $v$ and the speed of light $c, \gamma$ is the Lorentz factor of the particle, and $\delta=0.63[10]$.

All the energy loss mechanisms are effective only within the disk of size $2 h \simeq 300$ pc apart from synchrotron radiation and, more importantly, the rate of energy loss depends also on the average number density of the hydrogen atoms in the disk, for which we adopt $n_{\mathrm{H}}=0.9 \mathrm{~cm}^{-3}$. Such an average density corresponds to the surface density of $2 \mathrm{mg} / \mathrm{cm}^{2}$ which is approximately the effective grammage of the Galactic disk from observation [11]. The specific form of the energy loss rate is collected from [12-14]. We take into account also the adiabatic energy loss due to advection with the approximation $\left|\dot{E}_{a d}\right|=2 p v u_{0} \delta(z) \simeq p v u_{0} /(3 h)$ [15], $p$ denoting the particle momentum. As for the injection spectrum, we shall adopt the following power-law form in momentum down to the kinetic energy of $1 \mathrm{MeV}$ meaning $Q(E) \sim p^{2-\alpha} / \beta$ with $\alpha=4.23$ as suggested for the fit at 
high energies [10]. Even though the extension of the spectrum down to $1 \mathrm{MeV}$ seems questionable, there exists observational evidence of enhanced ionisation rates in the vicinity of SNRs indicating the presence of low-energy GCRs accelerated from these objects [16, 17].
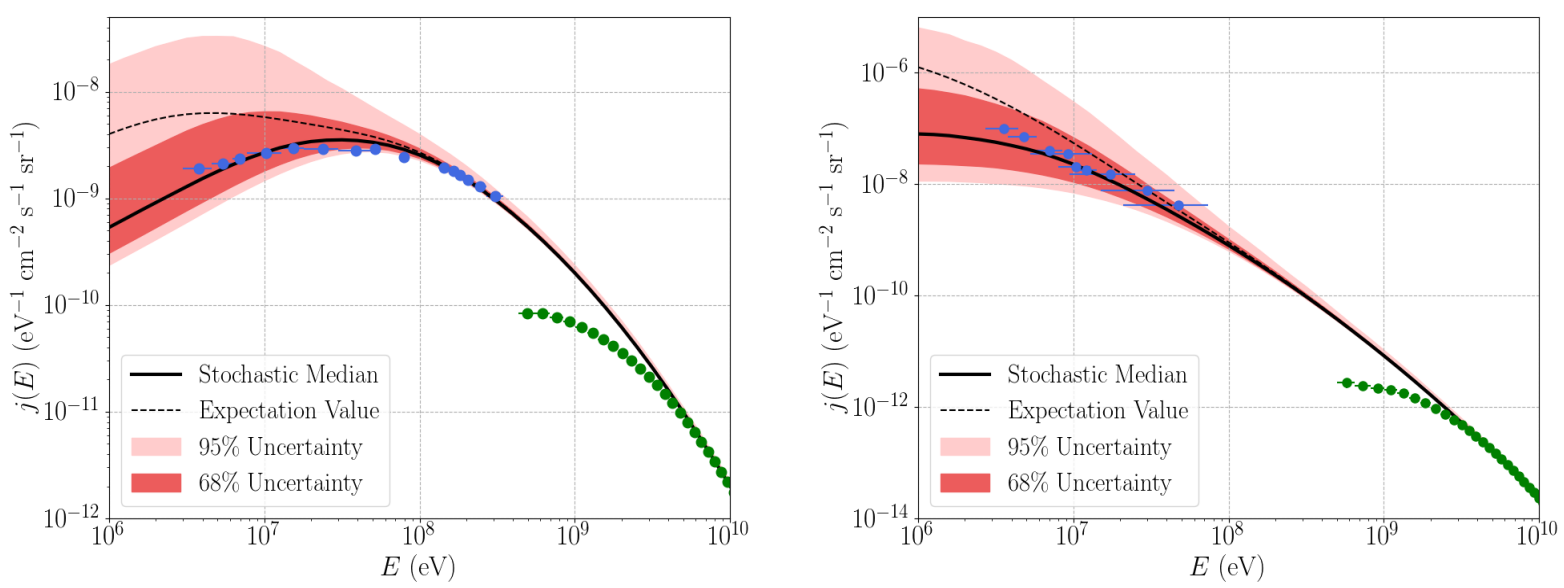

Figure 1: Stochastic fluctuations of GCR protons (left panel) and electrons (right panel) in comparison with data from Voyager [4] (blue) and AMS [18, 19] (green). The dotted and solid black curves are respectively the expectation values and the median of the intensities. The shaded regions are the $95 \%$ and $68 \%$ uncertainty ranges.

We have built up a statistical ensemble by generating a large number $N_{\mathrm{r}}=2000$ of realisations, in each drawing a large number of sources $N_{\mathrm{s}}$ from the spatial distribution following a spiral pattern with a radial modulation, as employed in [5], and with a homogeneous distributions for the time since injection and for the vertical position of sources. We limit ourselves to $r_{i}^{(n)}<r_{\max }=10$ $\mathrm{kpc}$ and the time since injection $\tau_{i}^{(n)}<\tau_{\max }=10^{8} \mathrm{yr}$ since older and further sources would not contribute significantly. The total number of discrete sources in each realisation could be estimated roughly as $N_{s}=\mathcal{R}_{s} \tau_{\text {max }} r_{\text {max }}^{2} / R_{d}^{2} \simeq 1.33 \times 10^{6}$, where $\mathcal{R}_{s} \simeq 0.03 \mathrm{yr}^{-1}$ is the source rate and $R_{d} \simeq 15 \mathrm{kpc}$ is the radius of the Galactic disk. We adopt also $2 h_{s} \simeq 80 \mathrm{pc}$ for the vertical extension of sources expected for core-collapse supernovae [20].

We thus obtain an ensemble of intensities $j^{(n)}=v /(4 \pi) \psi^{(n)}$ for the individual source realisations $n$ that we can characterise statistically. For instance, a histogram of these intensities at a specific energy could serve as an estimate of the intensity PDF. In the following, we call this $p(j)$. It is worth recalling that the expectation value of the intensity $\langle j\rangle=\int \mathrm{d} j p(j)$ is equal to the intensity predicted for the smooth source density of Ref. [5]. We have found that these distribution functions are not only asymmetric but they also do not have a well-defined second moment as has been shown for several analyses of the same type at high energies [5, 21]. We shall, therefore, specify the uncertainty intervals of the intensity using the percentiles as in [5], e.g. $j_{a} \%$ is defined via $a \%=\int_{0}^{j_{a} \%} \mathrm{~d} j p(j)$. The $68 \%$ and $95 \%$ uncertainty ranges of the intensity $j(E)$ are then $\mathcal{I}_{68 \%}=\left[j_{16 \%}, j_{84 \%}\right]$ and $\mathcal{I}_{95 \%}=\left[j_{2.5 \%}, j_{97.5 \%}\right]$. 


\section{Results and Discussion}

We present in Fig. 1 the $95 \%$ and $68 \%$ uncertainty bands of the intensities for both GCR protons (left panel) and electrons (right panel) in the energy range from $1 \mathrm{MeV}$ to about $10 \mathrm{GeV}$ together with the expectation values of the intensities and data from Voyager 1 [4] and AMS-02 [18, 19]. It is straightforward to see that above $100 \mathrm{MeV}$ the uncertainty ranges are quite narrow since the energy loss time and the diffusive escape time are sufficiently large such that the distribution of GCRs inside the Galactic disk becomes more or less uniform. We note that this will not remain true for GCR electrons of energy above $10 \mathrm{GeV}$ since the energy loss rate for these particles become increasingly larger in this energy range which will result in significant stochastic fluctuations [5].

The uncertainty ranges below $100 \mathrm{MeV}$ broaden at lower energies until a characteristic energy $E^{*}$ below which the ratio between the upper and lower edge of the uncertainty interval becomes constant. Such a feature emerges from the fact that $\mathcal{G}\left(r=0, z=z_{\odot}, E, r_{i}, z, z_{i}, \tau_{i}\right) \sim 1 /|\dot{E}|$ if the propagation time $\tau_{i}$ is much larger than the energy loss time $\left(\tau_{i} \gg \tau_{l}(E)=E /|\dot{E}|\right)$ which is easily fulfilled for particles of energy below a few tens of MeV. Since $\tau_{i}^{(n)} \gtrsim \tau_{l}(E \lesssim 10 \mathrm{MeV})$ for $i=\overline{1, N_{s}}$ in each of the $N_{r}$ realization, we expect from Eq. 2 that $j^{(n)}(E) \sim v /|\dot{E}|$ for all realizations at sufficiently low energies and, thus, the limits of the uncertainty ranges should become parallel below a characteristic energy. Since $\tau_{i}^{(n)} \gtrsim \tau_{l}(E \lesssim 10 \mathrm{MeV})$ for $i=\overline{1, N_{s}}$ in each of the $n$th realization, we expect from Eq. 2 that $j^{(n)}(E) \sim 1 /|\dot{p}|$ for all realizations at sufficiently low energies and, thus, the limits of the uncertainty ranges should become parallel below a characteristic energy. The intensities of GCR protons for several realizations which are within the $68 \%$ uncertainty range are depicted in Fig. 2 to better illustrate the spectral behaviour at low energies.
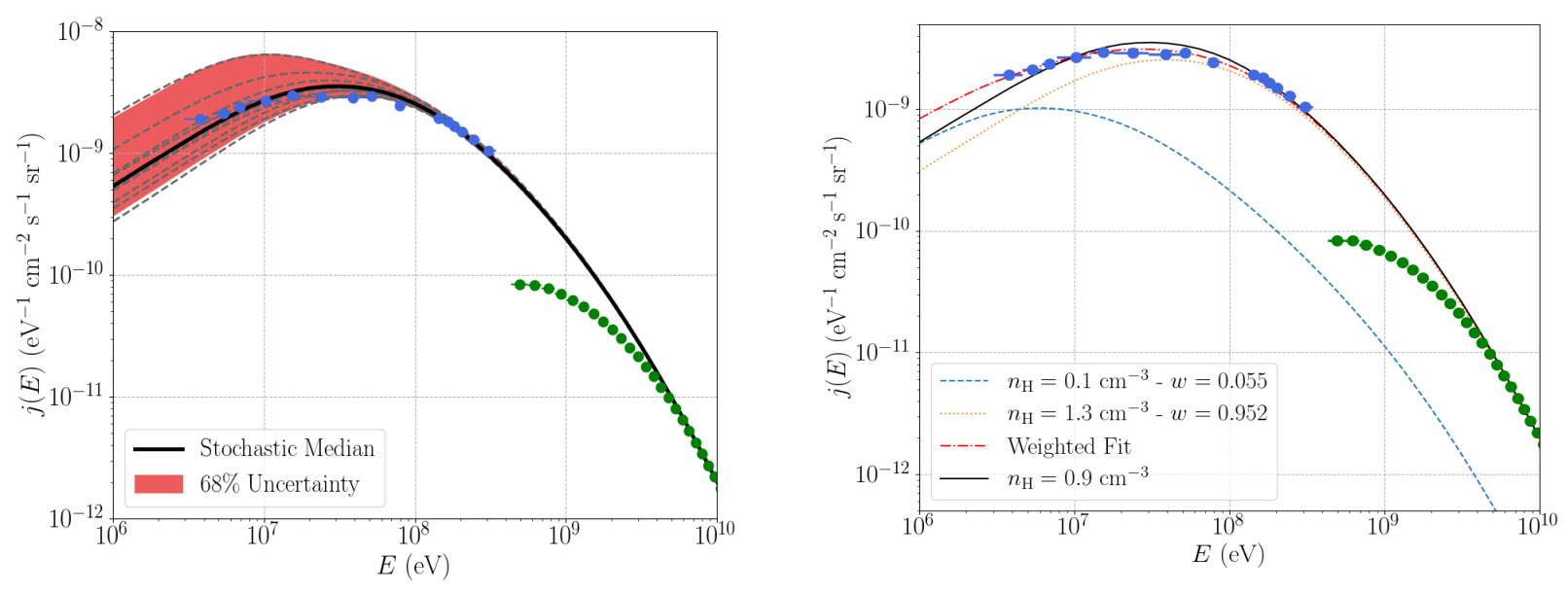

Figure 2: Left: Intensities of GCR protons for several realizations (dashed grey curves) around the $68 \%$ uncertainty range (shaded region). Data points are as in Fig. 1 and the solid black curve is the median of the intensities. Right: Weighted sum (red dot-dash curve) of the stochastic median in two cases $n_{\mathrm{H}}=0.1 \mathrm{~cm}^{-3}$ (dash blue curve) and $n_{\mathrm{H}}=1.3 \mathrm{~cm}^{-3}$ (dotted yellow curve) in comparison to the case for $n_{\mathrm{H}}=0.9 \mathrm{~cm}^{-} 3$ (black solid curve).

Note that a uniform distribution of GCRs will be attained if the number of sources within the 
diffusion loss length $l_{d}(E)=\sqrt{4 D(E) \tau_{l}(E)}$ in the disk is much larger than one,

$$
\mathcal{R}_{s} \tau_{l}(E) \frac{2 l_{d}^{3}(E)}{3 R_{d}^{2} h_{s}} \gg 1 .
$$

We can use this to estimate the characteristic energy $E^{*}$ by setting the LHS of the above inequality to one, which gives $E^{*} \simeq 10 \mathrm{MeV}$ for both GCR protons and electrons.

Interestingly, the median corresponding to $j_{50 \%}$, the $50 \%$ percentile of the PDF of the intensities, seems to provide a good fit to the data of Voyager and AMS for both GCR protons and electrons (see Fig. 1). We note that both the expectation values and the median do not strictly correspond the intensities of any particular realizations of sources. At low energies, however, the expectation value is dominated by a few, but rather unlikely realisations with extreme intensities such that $j^{(n)}(E)>$ $j_{84 \%}(E)$ which are outside of the $68 \%$ uncertainty range (as could be seen in Fig. 1). Furthermore, the resulting $\langle j(E)\rangle$, which is also the intensities predicted for the smooth source density as stressed above, has a different energy dependence than the universal scaling $j^{(n)}(E) \sim v /|\dot{E}|$ expected at low energies. The median, on the other hand, behaves as $j_{50 \%}(E) \sim v /|\dot{E}|$ and, in fact, the intensities in many realizations seem to closely resemble the spectral behaviour of the median both at low and high energies (see Fig. 2). It is for this reason that the median is to be preferred over the expectation value for the comparison with observational data.

We note also that the observed proton spectrum seems to have a broader peak than the median of the stochastic model and the observed electron spectrum seems to exceed the median. It is clear, however, that the local ISM should be quite inhomogeneous. Note that the observed spectra in such an inhomogeneous ISM could be modelled as the weighted average of spectra for different gas densities to provide better agreement with data. We have shown an example where the median of the proton intensities in two cases with $n_{\mathrm{H}}=0.1 \mathrm{~cm}^{-3}$ and $n_{\mathrm{H}}=1.3 \mathrm{~cm}^{-3}$ are weighted to provide a broadening of the peak and improve the fit to the Voyager data (see Fig. 2).

\section{Summary and outlook}

We have presented results of a modelling of proton and electron spectra between $1 \mathrm{MeV}$ and $10 \mathrm{GeV}$. Before the advent of the Voyager measurements outside the heliopause, this energy range had received relatively little attention due to the fact that solar modulation makes the inference of interstellar spectra difficult. All the models to date assume a smooth source distribution, however, these models do not reproduce the Voyager data unless a spectral break is introduced in the source spectrum. From a microphysical point of view, such a break seems rather unmotivated.

However, the smooth approximation is not justified since at low energies the energy loss distance becomes shorter than the average source separation. Unlike previous models we therefore considered the discrete nature of sources, modelling the distribution of intensities in a statistical ensemble. We noted that the intensity prediction from a smooth density is the ensemble average of this distribution. However, we showed that the ensemble average is not representative of the distribution due to its long power law tails. For instance, the spectral shapes of the predicted intensities in different realisations are the same below a critical energy. While the expectation value has a very different spectrum at the lowest energies, the median of the distribution does exhibit the same spectral shape. Furthermore, the expectation value is outside the $68 \%$ uncertainty range of 
the distribution at the lowest energies while the median is by definition always inside. We have shown that the Voyager 1 data fall squarely around the median of the distribution without the need for any unphysical breaks in the source spectrum.

The statistical model we have presented here might have interesting implications for other anomalies observed in low-energy GCRs. For instance, it has been shown recently [22] that the ionisation rate implied by the Voyager data is much smaller than the ionisation rate directly inferred for a large number of molecular clouds. It would be interesting to see whether the inhomogeneities implied by our statistical model of discrete sources can alleviate this tension. Thanks to our careful statistical model, we will be able to statistically quantify such a model in the future.

\section{Acknowledgments}

This project has received funding from the European Union's Horizon 2020 research and innovation programme under the Marie Skłodowska-Curie grant agreement No 665850. VHMP is grateful to Marco Kuhlen, Nhan Chau, and Ngoc Khanh Vu for fruitful discussions and technical support.

\section{References}

[1] S. Gabici, C. Evoli, D. Gaggero, P. Lipari, P. Mertsch, E. Orlando et al., The origin of Galactic cosmic rays: Challenges to the standard paradigm, International Journal of Modern Physics D 28 (2019) 1930022 [1903. 11584].

[2] M. S. Potgieter, Solar Modulation of Cosmic Rays, Living Reviews in Solar Physics 10 (2013) 3 [1306. 4421].

[3] E. C. Stone, A. C. Cummings, F. B. McDonald, B. C. Heikkila, N. Lal and W. R. Webber, Voyager 1 Observes Low-Energy Galactic Cosmic Rays in a Region Depleted of Heliospheric Ions, Science 341 (2013) 150.

[4] A. C. Cummings, E. C. Stone, B. C. Heikkila, N. Lal, W. R. Webber, G. Jóhannesson et al., Galactic Cosmic Rays in the Local Interstellar Medium: Voyager 1 Observations and Model Results, ApJ 831 (2016) 18.

[5] P. Mertsch, Cosmic ray electrons and positrons from discrete stochastic sources, J. Cosmology Astropart. Phys. 2011 (2011) 031 [1012 . 0805].

[6] J. P. Nolan, Univariate stable distributions: models for heavy tailed data. 2020.

[7] D. M. Skowron, J. Skowron, P. Mróz, A. Udalski, P. Pietrukowicz, I. Soszyński et al., A three-dimensional map of the Milky Way using classical Cepheid variable stars, Science 365 (2019) 478 [1806. 10653].

[8] V. H. M. Phan, F. Schulze, P. Mertsch, S. Recchia and S. Gabici, Stochastic Fluctuations of Low-Energy Cosmic Rays and the Interpretation of Voyager Data, arXiv e-prints (2021) arXiv:2105.00311 [2105.00311]. 
[9] R. Schlickeiser, M. Lazar and M. Vukcevic, The Influence of Dissipation Range Power Spectra and Plasma-wave Polarization on Cosmic-ray Scattering Mean Free Path, ApJ 719 (2010) 1497.

[10] C. Evoli, R. Aloisio and P. Blasi, Galactic cosmic rays after the AMS-02 observations, Phys. Rev. D 99 (2019) 103023 [1904 . 10220].

[11] K. M. Ferrière, The interstellar environment of our galaxy, Reviews of Modern Physics 73 (2001) 1031 [astro-ph/0106359].

[12] R. Schlickeiser, Cosmic Ray Astrophysics. 2002.

[13] S. Krakau and R. Schlickeiser, Pion Production Momentum Loss of Cosmic Ray Hadrons, ApJ 802 (2015) 114.

[14] C. Evoli, D. Gaggero, A. Vittino, G. Di Bernardo, M. Di Mauro, A. Ligorini et al., Cosmic-ray propagation with DRAGON2: I. numerical solver and astrophysical ingredients, J. Cosmology Astropart. Phys. 2017 (2017) 015 [1607. 07886].

[15] É. Jaupart, É. Parizot and D. Allard, Contribution of the Galactic centre to the local cosmic-ray flux, A\&A 619 (2018) A12 [1808.02322].

[16] S. Vaupré, P. Hily-Blant, C. Ceccarelli, G. Dubus, S. Gabici and T. Montmerle, Cosmic ray induced ionisation of a molecular cloud shocked by the W28 supernova remnant, A\&A 568 (2014) A50 [1407.0205].

[17] V. H. M. Phan, S. Gabici, G. Morlino, R. Terrier, J. Vink, J. Krause et al., Constraining the cosmic ray spectrum in the vicinity of the supernova remnant W28: from sub-GeV to multi-TeV energies, A\&A 635 (2020) A40 [1910.09987].

[18] M. Aguilar et al. (AMS Collaboration), Precision Measurement of the $\left(e^{+}+e^{-}\right)$Flux in Primary Cosmic Rays from $0.5 \mathrm{GeV}$ to $1 \mathrm{TeV}$ with the Alpha Magnetic Spectrometer on the International Space Station, Phys. Rev. Lett. 113 (2014) 221102.

[19] M. Aguilar et al. (AMS Collaboration), Precision Measurement of the Proton Flux in Primary Cosmic Rays from Rigidity 1 GV to 1.8 TV with the Alpha Magnetic Spectrometer on the International Space Station, Phys. Rev. Lett. 114 (2015) 171103.

[20] N. Prantzos, C. Boehm, A. M. Bykov, R. Diehl, K. Ferrière, N. Guessoum et al., The 511 keV emission from positron annihilation in the Galaxy, Reviews of Modern Physics 83 (2011) 1001 [1009. 4620].

[21] Y. Genolini, P. Salati, P. D. Serpico and R. Taillet, Stable laws and cosmic ray physics, A\&A 600 (2017) A68 [1610.02010].

[22] V. H. M. Phan, G. Morlino and S. Gabici, What causes the ionization rates observed in diffuse molecular clouds? The role of cosmic ray protons and electrons, MNRAS 480 (2018) 5167 [1804 . 10106]. 